\title{
PREPARATION AND LABELING OF TECHNITIUM-99M KIT IN PHARMACEUTICAL GRADE CLEAN ROOM
}

\author{
${ }^{1}$ Ruhul Amin M*., ${ }^{2}$ Azizul Haque, ${ }^{1}$ Avishek Biswas and ${ }^{1}$ Taufiq Hassan Mozumder \\ ${ }^{I}$ Department of Chemical Engineering, Bangladesh University of Engineering \& Technology (BUET), \\ Dhaka-1000, Bangladesh \\ ${ }^{2}$ Chief Scientific Officer and Head Radioisotope Production Division (RIPD) \\ Institute of Nuclear Science and Technology, Atomic Energy Research Establishment, Savar, Dhaka
}

\begin{abstract}
Technetium-99m radiopharmaceuticals are in widespread use owing to the availability and affordability of $99 \mathrm{Mo} / 99 \mathrm{~m}-\mathrm{Tc}$ generators and the variety of kits for formulating the desired products. Together, they provide an array of specific tools for diagnosing a large number of diseases affecting the bones and major organs of the body such as the heart, brain, liver, kidney and thyroid. Nuclear medicine requires high quality radiopharmaceuticals and kits that are safe for administration and efficacious for a given application. In Bangladesh, no commercial production was done before this. This paper presents the theoretical basis of and describes the procedures for preparing three selected kits. Details of the ingredients are also included. The procedures described here can be used to develop manuals and standard operating procedures in Bangladesh. This report is expected to serve as a guide to radiopharmaceutical manufacturing centers and centralized pharmacies involved in the production of such kits in the current environment of Bangladesh. It will be a useful resource for many hospital radio pharmacy departments that routinely use the kits to compound 99m-Tc radiopharmaceuticals. Three kits namely: MDP, DMSA and DTPA were prepared. Paper Chromatography was involved to disparate the polar and non-polar part of the kit-solution and finally gamma spectrometry (HPGe) to deduce the Radiochemical Purity (RCP). By reviewing purity values, it was found that only DMSA has passed the $95 \%$ barrier which is the required percentage for safe application. This paper suggests that the findings require more practice and involve such exquisite points, which will contribute in the production of Tc-99m vivo-kits at Radioisotope Production Division (RIPD), AERE, INST and in their projected future mass production successfully.
\end{abstract}

\section{INTRODUCTION}

Nuclear medicine is a medical specialty involving the application of radioactive substances in the diagnosis and treatment of diseases. In nuclear medicine procedures, radio-nuclides are combined with other elements to form chemical compounds, or else combined with existing pharmaceutical compounds to form radiopharmaceuticals. ${ }^{(1)}$

Radio pharmacology is the study and preparation of radio pharmaceuticals. Radiopharmaceuticals have been defined as radioactive drugs that, when used for the purpose of diagnosis or therapy, typically elicit no physiological response from the patient. This definition is strongly supported by the Nuclear Medicine community's collective experience in administering radiopharmaceuticals. ${ }^{(2)}$ The design of these compounds is based solely upon physiological function of the target organ.

The mechanism of localization of a radiopharmaceutical in a particular target organ can be as simple as the physical trapping of particles or as sophisticated as an antigen-antibody reaction or chemisorption of an inorganic phosphate on the hydroxyapatite crystals deposited in an acute myocardial infarction.

The term radioisotope has historically been used to refer to all radiopharmaceuticals, and this usage remains common. Technically, however, many radiopharmaceuticals incorporate a radioactive tracer atom into a larger pharmaceutically-active molecule, which is localized in the body, after which the radionuclide tracer atom allows it to be easily detected with a gamma camera or similar gamma imaging device. Some radioisotopes (for example gallium-67, gallium-68, and radioiodine) are used directly as soluble ionic salts, without further modification. This use relies on the chemical and biological properties of the radioisotope itself, to localize it within the body.

The growth of nuclear medicine has been due mainly to the availability of ${ }^{99 \mathrm{~m}} \mathrm{Tc}$ radiopharmaceuticals; this single isotope is used in over $80 \%$ of all diagnostic procedures. Each year, roughly 25 million procedures are carried out with ${ }^{99 \mathrm{~m}} \mathrm{Tc}$ radiopharmaceuticals, and this figure is projected to grow at a rate of about $15 \%$ per annum. The availability of short lived ${ }^{99 \mathrm{~m}} \mathrm{Tc}$ (halflife: $6 \mathrm{~h}$ ) from the ${ }^{99} \mathrm{Mo} /{ }^{99 \mathrm{~m}} \mathrm{Tc}$ generator, as the 
daughter product of long lived ${ }^{99}$ Mo (half-life: $67 \mathrm{~h}$ ), is a major factor behind the universal use of this radioisotope. The parent radionuclide, ${ }^{99} \mathrm{Mo}$, is prepared in abundant quantities by the fission of ${ }^{235} \mathrm{U}$ in a nuclear reactor, with a fission yield of $6 \%$. There are several major producers that collectively have the capacity to produce enough ${ }^{99}$ Mo to meet current global demand.

Technetium-99m radiopharmaceuticals are generally formulated from kits prepared in authorized manufacturing facilities. A cold kit contains the ligand to which ${ }^{99 \mathrm{~m}} \mathrm{Tc}$ is to be complexed, an adequate quantity of reducing agent, buffer to adjust the $\mathrm{pH}$ to suit the labeling conditions, stabilizing agents and excipients. The kits are prepared in a freeze-dried form and have a long shelf life, ranging from several months to years. The kits can be transported at room temperature; however, storage in a refrigerator at $2-8^{\circ} \mathrm{C}$ is advantageous and ensures a long shelf life in most cases.

The compounding of $99 \mathrm{mTc}$ radio-pharmaceuticals using kits is fairly easy, as it involves the addition of $99 \mathrm{~m} \mathrm{TcO}^{4-}$ eluted from a generator, generally at room temperature but at times with heating. The use of chromatographic techniques such as paper chromatography, Instant Thin Layer Chromatography (ITLC) or High Performance Liquid Chromatography (HPLC) is recommended for estimation of the radiochemical purity of the final product, prior to administration to patients. Radiopharmaceuticals are considered to be compounded sterile products, and it is recommended that compounding of $99 \mathrm{mTc}$ radiopharmaceuticals be carried out in an ISO 5 (class 100, grade A) laminar flow bench located in a clean room (with a buffer zone).

\section{EXPERIMENTAL}

Materials: We worked on three kits in Atomic Energy Research Establishment; those are DTPA, DMSA and MDP. These kits are briefly discussed below.

MDP: All 99m Tc-phosphonates with ligands such as MDP can be characterized by the general formula $\mathrm{H}_{2} \mathrm{O}_{3} \mathrm{P}-\mathrm{X}-\mathrm{PO}_{3} \mathrm{H}_{2}$, where $\mathrm{X}=-\mathrm{CH}_{2}$ for MDP. Independent of the substituents, phosphonates show a tendency to form oligomers, which can be avoided by adding antioxidants such as ascorbic acid prior to freeze-drying the product. Their main field of application is bone scintigraphy, because technetium phosphonates are taken up by normal bone and bone lesions by chemisorption, followed by exchange on the hydroxyapatite, the inorganic matrix of the bone. Higher uptake is observed when the regional blood flow is higher owing to hydroxyapatite formation accompanied by increased osteoblastic activity. Thus 2-8 times the activity of technetium phosphonates can accumulate in bone lesions with increased osteoblastic activity (rupture, tumor metastases, etc.) compared with normal bone.

DMSA: In an alkaline medium ( $\geq \mathrm{pH} 8)$, all of the free -SH groups of Di-Mercapto-Succinic Acid (DMSA) are ready to react with technetium, forming a penta coordinated bis-complex, 99m Tc-DMSA (V). This bis-complex accumulates both in the kidneys and in soft tissue tumors such as medullar carcinomas. If the $\mathrm{pH}$ does not exceed 9, the compound is of an appropriate stability and is injectable. When labeling of DMSA is performed in acidic media, a hexacoordinated asymmetric bis-complex is formed in which one molecule is bound to technetium via two $\mathrm{S}$ - bridges and one -O- bridge, while the other is bound via one -S- bridge and two -O- bridges, and one $-\mathrm{SH}$ remains free. This kind of structure of $99 \mathrm{~m}$ Tc-DMSA (III) is complete if the $\mathrm{pH}$ is around 3. The asymmetric complex, when injected, is taken up by the kidneys and remains bound owing to a ligand exchange reaction occurring between the DMSA ligand and the protein located in the proximal tubules of the kidney.

Complete uptake of $99 \mathrm{~m}$ Tc-DMSA (III) is observed $2-4 \mathrm{~h}$ post-injection. Renal scintigraphy using $99 \mathrm{~m}$ Tc-DMSA (III) provides quantitative information about the functional mass of each individual kidney, since the renal uptake is proportional to the functional mass.

DTPA: A well-known radiopharmaceutical with a hydrophilic character is $99 \mathrm{~m}$ Tc-DTPA. However, only up to $10 \%$ of the $99 \mathrm{~m}$ Tc-DTPA is bound to plasma proteins, and almost complete glomerular excretion occurs via the kidneys. More than $90 \%$ of the injected activity is washed out within $2 \mathrm{~h}$ for $99 \mathrm{~m}$ Tc-DTPA. Other applications of $99 \mathrm{~m}$ Tc-DTPA include blood flow studies (brain, heart, and extremities), cerebrospinal fluid circulation studies, studies of transport in the gastrointestinal tract with labeled drinks and foods, and inhalation studies using 99m Tc-DTPA aerosol.

\section{PROCEDURES}

Various Tc-99m radiopharmaceuticals (MDP, DMSA, DTPA) including pertechnetate, Tc-99m sulfur colloid, Tc-99m Sn MAA, Tc-99m Sn phytate, and Tc-99m stannous chloride were each spotted on the bottom pencil line of strips of whatman no.1 chromatography. The strips were promptly placed in testtube, to which approximately $1 \mathrm{ml}$ of MEK was 
added and developed until the solvent front had migrated to the top pencil line (approximately $10 \mathrm{~min}$.). The strips were dried and cut into equal segments: below and above the center line. Each segment was then counted for activity. Same procedures have been done with the saline solution. Tc-99mSn DMSA was spotted on the bottom pencil line of ten Whatman no.1 paper strips,developeding into MEK (approximately 15min.), sectioned and counted similarly. Two samples for both batch one and two were performed on each agent.

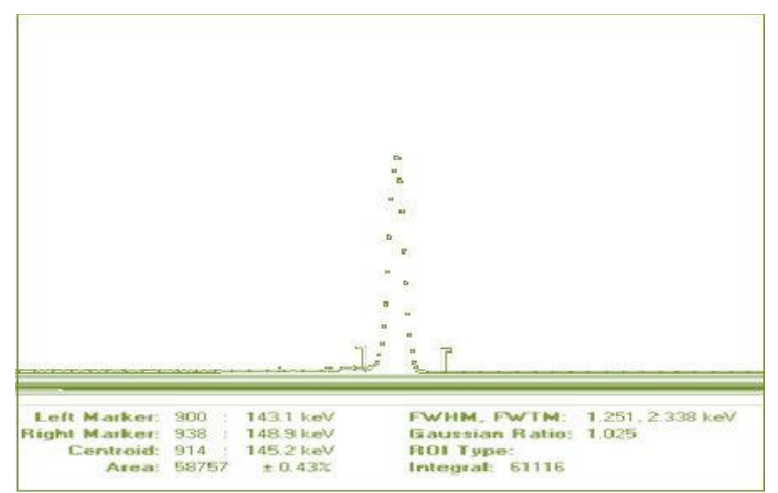

Fig 01: DMSA-iii Batch-2 sample-2 Top reading in HPGe detector

Each determination consisted of spotting the specific radiopharmaceutical on Whatman no.1 paper strips on the bottom pencil line. The Whatman no.1 paper were immediately placed in a test tube containing approximately $1 \mathrm{ml}$ of MEK and developed until the solvent front migrated to the top pencilline (about $10 \mathrm{~min}$.). Both strips were then removed,cut into four segmentsabove and four below the center line, and each segment counted for activity using gamma spectrometry (HPGe).

\section{Data Analysis and Results}

For MDP Kit:

pH: 6-7,

Activity: $10 \mathrm{~mL}$ saline: $155.5 \mathrm{mCi}$

For $5 \mathrm{mCi} 0.3 \mathrm{~mL}$ solution needed

Stationary Phase: Whatman no. 1

Mobile Phase: MEK (Methyl Ethyle Ketone)
Table 1: Top and Bottom reading from HPGe detector for MEK solution

\begin{tabular}{|l|l|l|l|}
\hline Batch & Sample & Top (A) & Bottom (B) \\
\hline 01 & 01 & 177 & 12246 \\
\hline 01 & 02 & 1191 & 23624 \\
\hline 02 & 01 & 438 & 43317 \\
\hline 02 & 02 & 367 & 23615 \\
\hline
\end{tabular}

Stationary Phase: Whatman no. 1

Mobile Phase: Saline

Table 2: Top and Bottom reading from HPGe detector for Saline $\left(\mathrm{NaCl}+\mathrm{NaNO}_{3}\right)$ solution

\begin{tabular}{|l|l|l|l|}
\hline Batch & Sample & Top (B) & Bottom (A) \\
\hline 01 & 01 & 16624 & 4511 \\
\hline 01 & 02 & 10881 & 4157 \\
\hline 02 & 01 & 17720 & 5918 \\
\hline 02 & 02 & 41744 & 10818 \\
\hline
\end{tabular}

For DMSA Kit:

Stationary Phase: Whatman no. 1

Mobile Phase: MEK (Methyl Ethyle Ketone)

pH: 3.5-4.0

Batch: 1

Activity: $119.3 \mathrm{mCi}$ for saline

For $5 \mathrm{mCi} 0.42 \mathrm{~mL}$ solution needed

Table 3: Top and Bottom reading from HPGe detector for MEK Solution

\begin{tabular}{|c|l|l|l|}
\hline Batch & Sample & Top (B) & Bottom (A) \\
\hline 1 & 01 & 13439 & 656 \\
\cline { 2 - 4 } & 02 & 26556 & 1285 \\
\hline 2 & 01 & 8348 & 1101 \\
\cline { 2 - 4 } & 02 & 58757 & 7224 \\
\hline
\end{tabular}


$2^{\text {nd }}$ Evaluation:

$\mathrm{pH} \leq 3.5$

Stationary Phase: Whatman no. 1

Mobile Phase: Butanol : Acetic Acid : Water

Table 4: Top and Bottom reading from HPGe detector for Butyl Acetic Acid solution

\begin{tabular}{|l|l|l|l|}
\hline Batch & Sample & Top (A) & Bottom (B) \\
\hline 01 & 01 & 23050 & 5164 \\
\hline 02 & 01 & 20060 & 4560 \\
\hline
\end{tabular}

For DTPA Kit:

Stationary Phase: Whatman no. 1

Mobile Phase: MEK (Methyl Ethyl Ketone)

$\mathrm{pH}: 4.5-5$

Activity: $9.58 \mathrm{mCi}$ for saline solution

For $5 \mathrm{mCi}$ solution $5.22 \mathrm{~mL}$ solutions needed

Table 5: Top and Bottom reading from HPGe detector for MEK solution

\begin{tabular}{|l|l|l|l|}
\hline Batch & Sample & Top (A) & Bottom (B) \\
\hline 01 & 01 & 5392 & 75034 \\
\hline 01 & 02 & 17212 & 29874 \\
\hline 02 & 01 & 2974 & 150169 \\
\hline 02 & 02 & 3142 & 28370 \\
\hline
\end{tabular}

Stationary Phase: Whatman no. 1

Mobile Phase: Saline

Table 6: Top and Bottom reading from HPGe detector for Saline $\left(\mathrm{NaCl}+\mathrm{NaNO}_{3}\right)$ solution

\begin{tabular}{|l|l|l|l|}
\hline Batch & Sample & Top (B) & Bottom (A) \\
\hline 01 & 01 & 72774 & 8516 \\
\hline 01 & 02 & 27228 & 4570 \\
\hline 02 & 01 & 8646 & 6673 \\
\hline 02 & 02 & 7027 & 9693 \\
\hline
\end{tabular}

This final table illustrates all our findings in RCP values for different solutions used on different kit preparation.
Table 7: The radio-chemical purity for the produced kit as calculated

\begin{tabular}{|c|c|c|c|c|c|}
\hline Kit & Batch & $\begin{array}{l}\text { Sample } \\
\text { no. }\end{array}$ & $\begin{array}{l}\mathrm{RCP} \text { in } \\
\mathrm{MEK} \%\end{array}$ & $\begin{array}{l}\mathrm{RCP} \text { in } \\
\text { Saline\% }\end{array}$ & $\begin{array}{l}\text { Total } \\
\text { RCP\% }\end{array}$ \\
\hline \multirow{4}{*}{$\hat{\mathrm{E}}$} & 1 & 1 & 98.57 & 78.66 & 77.24 \\
\hline & & 2 & 95.2 & 72.26 & 67.57 \\
\hline & 2 & 1 & 99 & 74.96 & 73.96 \\
\hline & & 2 & 98.47 & 74.58 & 73.06 \\
\hline \multirow{4}{*}{$\sum_{0}^{\overleftarrow{\Delta}}$} & 1 & 1 & 95.34 & - & 95.34 \\
\hline & & 2 & 95.38 & - & 95.38 \\
\hline & 2 & 1 & 88.35 & - & 88.35 \\
\hline & & 2 & 89.05 & - & 89.05 \\
\hline \multirow{4}{*}{$\begin{array}{l}\mathbb{\nwarrow} \\
\text { 合 }\end{array}$} & 1 & 1 & 93.29 & 10.47 & 82.81 \\
\hline & & 2 & 63.44 & 85.62 & 49.07 \\
\hline & 2 & 1 & 98.05 & 56.43 & 54.48 \\
\hline & & 2 & 90.02 & 57.98 & 32.05 \\
\hline
\end{tabular}

\section{DISCUSSION}

This paper provides every nuance of plausible experimental data to represent all knotty aspects of Tc-99m kit production for a certain environment. It is required that the percentage of purity in the mobile phase be equal or over $95 \%$ for each vivo-kit. We prepared three kits (MDP, DTPA and DMSA) and tested into three solutions (MEK, Saline and Butyle Acetic Acid) for a result replete to remove all error due to pertechnetate and hydrated technetium.

Data analysis has brought us to a rather enigmatic conclusion, whereas; only DMSA kit has passed that 95\% purity barrier in Methyl Ethyle Ketone solution as mobile phase. MDP kits were over $78 \%$ pure and DTPA about $82 \%$. It is obvious that the expecting result has repudiated its course for reasons related to the experimental procedures and timeline. We will consider every credible reason that could have engendered this tangency.

It is somewhat confounding to ensconce to a belief which must be the sole reason that caused our experimental data to rescind its way from success. But time value for all radio-pharmaceuticals must act 
as a bolster for any case of contamination. We've been working on this project for about six months continuously and produced kits from time to time. This time ranges from 2-3 months for some batches. It is obvious that radioactive products are ephemeral unless freeze dried correctly. But in our absence; for some oblivious reason MDP kits were exposed to higher temperature (instead of $2-8^{\circ} \mathrm{C}$ ). This brought the kits to a solution form for a period of time. It could be a tentative reason to disregard our experimental faults; yet fathomable. The following passage states why we should consider this as a lethal reason for some cases.

The cold kits used for preparation of $99 \mathrm{mTc}$ labelled radiopharmaceuticals contain one of the stannous salts as a reducing agent for converting technetium in the +7 state in the pertechnetate to a desired lower oxidation state, which complexes with the ligand to form the radiopharmaceutical. Stannous chloride dihydrate $\left(\mathrm{SnCl}_{2} \cdot 2 \mathrm{H}_{2} \mathrm{O}\right)$ is the most commonly used stannous salt. Stannous ions are highly prone to oxidation, even on exposure to atmosphere or dissolved oxygen in the solution. Hence, great care must be taken to perform kit formulations using solutions bubbled with nitrogen and to carry out the dispensing procedure as quickly as possible to minimize exposure to atmosphere. The presence of stannous ions in the kit vial is essential for preparation of the radiopharmaceutical, and in some cases the amount of stannous content present is crucial to obtaining the desired product quality. The stability of the kits depends on the continuous presence of the stannous content throughout the period, which in turn depends on, for example, the production process, freeze-drying conditions and residual humidity, as well as the storage conditions must be placed, however, on cost effectiveness in order to abolish the argument that procedures are too expensive and to demonstrate the innocuousness of the low dose radiation used in most procedures.
Universities, nuclear medicine societies and associations, commercial companies as well as international institutions like the IAEA are expected to play a major role regarding all the above mentioned aspects. Linking the key professional bodies of nuclear medicine with the IAEA and the use of electronic journals should improve the communication between practitioners of nuclear medicine, especially in developing countries like Bangladesh.

\section{CONCLUSION}

This work investigates over different procedures. We have worked our way up until now to find out better ways to produce ${ }^{99 \mathrm{~m}}$ Tc kits. We stayed headstrong and finally came to this tremendous feat. Our works has proved that there are ways to improve Tc-99m radio-pharmaceuticals in our country alongside the various conditions and atmosphere that we have to face. Not to sound as a plaudit; but this report has provided some noteworthy facts for 'what future holds in Bangladesh radio-pharmacy'.

\section{References}

[1] HENKIN R. et al: Nuclear Medicine. First edition (1996).

[2] SCHWOCHAU, KLAUS. Technetium. Wiley-VCH (2000)

[3] DR. ABEDIN, MD. ZAINUL. Diagnostic and Therapeutic Radiopharmaceuticals , (2009)

[4] EUROPEAN COMMISSION, EU Guidelines to Good Manufacturing Practice, EUDRALEX, Vol. 4, Medicinal Products for Human and Veterinary Use: Good Manufacturing Practice, EU, Luxembourg (2003) 